\title{
«Datificación» y Educación Superior: Hacia la construcción de un marco para la alfabetización en datos del profesorado universitario
}

\author{
Juliana E. Raffaghelli ${ }^{1}$
}

Recibido: $11-05-2019$

Aceptado: 10-09-2019

\section{Resumen}

La «datificación» de procesos y servicios es un fenómeno emergente, que está creando una nueva forma de divisoria digital (Kitchin, 2014). La abundancia de datos generados y disponibles a partir del uso aplicativos web y móviles requiere competencias específicas para su explotación; además, han surgido nuevas preocupaciones, como la vigilancia y la incapacidad de comprender el poder y el control detrás del seguimiento de datos y las características de los algoritmos aplicados para el uso de dichos datos (Kennedy, Poell y van Dijck, 2015). La alfabetización en datos (Data Literacy) en general y de los educadores en particular serían un medio para lograr la apropiación activa y crítica de la riqueza provista por los datos, frenando inclusive las aristas más negativas del fenómeno (Dunlap y Piro, 2016; Markham, 2018 y Raffaghelli, 2017a y 2018a). Este artículo tiene como objetivo introducir un marco conceptual que apoye la alfabetización en datos del profesorado universitario, para capacitarlos en relación al fenómeno de la sociedad "datificada». El estudio se basa en una revisión de la literatura y es parte de una etapa inicial de un proyecto de investigación más amplio. Los marcos de alfabetización en datos, incluido el marco europeo 
DigCompEdu (Redecker y Punie, 2017) que ha sido adoptado para orientar la formación a la competencia digital de los educadores e incluye la alfabetización en datos, se analizan y discuten, con el objetivo de crear una síntesis de los componentes básicos y sus implicaciones para la investigación y la práctica.

Palabras clave: Alfabetización en datos, Desarrollo profesional de profesorado, DIGCOMPEDU.

\title{
«Datification» and Higher Education: Towards the construction of a framework for data literacy of university teaching staff
}

\begin{abstract}
The datification of processes and services is an emerging phenomenon, which is creating a new form of digital divide (Kitchin, 2014). The abundance of data generated and available from the web and mobile applications requires specific skills for their exploitation. In addition, new concerns have arisen, such as the problem of surveillance and the inability to understand the power and control behind data tracking and the characteristics of the algorithms applied to exploit such data (Kennedy, Poell, \& van Dijck, 2015). Data literacy in general and for educators in particular would be a means to achieve active and critical appropriation of the wealth provided by the data, curbing even the most negative edges of the phenomenon (Dunlap \& Piro, 2016; Markham, 2018; Raffaghelli, 2017, 2018). This paper aims at introducing a conceptual framework supporting educators' data literacy to empower them as education professionals, as well as their students, to thrive in the datafied society. The study is based on a review of the literature and it is part of an initial stage of a broader research project. Data literacy frameworks, including the DIGCOMPEDU Redecker
\end{abstract}


\& Punie, 2017) as framework for educators' digital competence which includes data literacy, are analyzed and discussed, aiming at creating a synthesis of basic components and its implications for research and practice.

Keywords: Data Literacy, Faculty Development, DIGCOMPEDU.

\section{Introducción}

Los datos digitalizados han entrado en nuestras vidas de manera masiva en los últimos diez años. Más allá de Internet en la era de la sociedad de la información, estamos presenciando el surgimiento de una sociedad "datificada», donde grandes cantidades de datos digitales, el ADN de la información, están liderando nuevas prácticas sociales. En efecto, los discursos más entusiastas sobre la abundancia de datos han enfatizado la oportunidad de generar nuevos modelos de negocios, nuevos escenarios profesionales relacionados con la ciencia de datos y prácticas abiertas en la ciencia y en el espacio público (EMC Education Services, 2015 y Scott, 2014). Más recientemente, la lógica bastante ingenua de la captura y articulación de datos a través de varios algoritmos como impulsores de formas de innovación económica y social como la selección de trabajadores, los incentivos a maestros por su práctica profesional o la inclusión en listas de espera para el otorgamiento de créditos hipotecarios, ha sido objeto de crítica y deconstrucción (Kitchin, 2015; O'Neil, 2016). En este escenario, la universidad como institución ha sido arrastrada a procesos de «datificación» de manera más bien forzada, en su esfuerzo por sobrevivir a una crisis de credibilidad, a través de formas de innovación ligadas a la digitalización de procesos y servicios (Daniel, 2015). Inicialmente, hubo intensos discursos que apoyaron las prácticas basadas en macrodatos (Big Data) como una oportunidad para mejorar la eficiencia, la objetividad, la transparencia y la innovación (Daniel, 2017). Sin embargo, hoy en día en este ámbito han surgido también nume- 
rosas críticas y preocupaciones respecto de cómo la «datificación» podría implicar, por ejemplo, un uso poco ético de los datos de estudiantes y docentes. Parece necesario, hoy más que nunca, generar intervenciones formativas para el profesorado y los estudiantes, que permitan elevar la toma de conciencia, aumentar habilidades técnicas y orientar nuevas prácticas en la relación entre estructuras digitales «datificadas», hacia formas de activa y crítica apropiación de la tecnología emergente.

\section{Estado del arte: «Datificación» en la educación superior y necesidad de impulsar nuevos marcos de alfabetización en datos}

Dos de las tres misiones principales de la educación superior (enseñanza e investigación, siendo la tercera extensión) han experimentado varios procesos de digitalización que abarcan prácticas de uso intensivo de datos (Daniel, 2017). Por el lado de la enseñanza, se están recopilando datos sobre el aprendizaje y la didáctica en una escala sin precedentes, dando lugar a la minería de datos educativos $y$, en particular, a las analíticas de aprendizaje (Ferguson, 2012; Nunn, Avella, Kanai y Kebritchi, 2016). Si bien no hay duda sobre el valor de las analíticas de aprendizaje para respaldar las prácticas pedagógicas de los docentes y la autorregulación de los estudiantes (Roll y Winne, 2015), las suposiciones sobre el poder de los algoritmos para predecir, apoyar o mejorar los procesos educativos pueden convertirse en prácticas de discriminación y vigilancia sin un adecuado control (Perrotta y Williamson, 2018). En general, las revisiones de la literatura han señalado vínculos deficientes entre los modelos de analíticas de aprendizaje y las teorías pedagógicas (Knight, Buckingham Shum y Littleton, 2014; Nunn et al., 2016), la falta de evaluación en contextos auténticos y la pobre aceptación por parte de profesores y alumnos (Vuorikari et al., 2016), sin mencionar los problemas sociales y éticos (Prinsloo y Slade, 2017; Slade y Prinsloo, 2013). Además, la adopción masiva de redes sociales imbricada con sistemas de gestión de aprendiza- 
je (Learning Management Systems-LMS) implicarían nuevas formas de recabado de datos que los profesores y los estudiantes pueden desconocer por completo (Manca, Caviglione y Raffaghelli, 2016).

Por el lado de la investigación, en cambio, el contexto digital, abierto y en red ha generado una gran cantidad de oportunidades para el desarrollo profesional, pero también ha incluido aspectos muy críticos sobre lo que se muestra y lo que se esconde en nuevos procesos de visibilidad digital (Stewart, 2013, 2015). Por otra parte, el paradigma de la ciencia abierta se basa en esquemas participativos en los que los ciudadanos están invitados a explorar y contribuir más estrechamente en todo el ciclo de recolección de datos, y requieren nuevas formas de pensar el propio hacer ciencia (Raffaghelli y Manca, 2019). De hecho, la apertura y reusabilidad de los datos de investigación podría acelerar la colaboración científica y el descubrimiento en formas sin precedentes. En el entrecruce entre estos dos mundos en fuerte cambio, la práctica académica podría potencialmente abordar nuevas conexiones entre la investigación y la enseñanza mediante el uso de datos educativos como contenido y para el avance de la enseñanza como parte de la ciencia educativa. El primer caso se basa en el concepto de datos abiertos como un recurso educativo abierto (Atenas, Havemann y Priego, 2015) en una cultura ampliamente científica. El segundo caso está relacionado con el movimiento general de la ciencia abierta aplicada a la investigación educativa (Zee y Reich, 2018). A pesar de tales escenarios prometedores para prácticas basadas en datos, la implementación de estas innovaciones en investigación y enseñanza implica tanto la reflexión profesional como la necesidad de un enfoque crítico (Raffaghelli y Manca, 2019; Zee y Reich, 2018).

Para la efectiva generación de prácticas basadas en datos en la educación superior, este escenario complejo requiere no solo la alfabetización técnica, sino también la alfabetización crítica, en el sentido de colocar los procesos de «datificación» (formas de recogida de datos, infraestructuras, algoritmos, ambientes digitales, visualización y circulación de datos) en contextos culturales y sociales. En particular, se trata de ir más allá de las habilidades de la elaboración estadística y de visualización de datos, dirigiéndose 
más bien hacia la capacidad de contextualizar e impulsar políticas de los datos producidos, manejados y utilizados con fines sociales y políticos (Raffaghelli, 2018b). En el caso de los datos académicos, la alfabetización de datos puede estar conectada a nuevas formas de profesionalidad que guían la investigación, la enseñanza y el aprendizaje en contextos digitales. De acuerdo con el conocido modelo de profesionalidas académica DIAT (Discovery, Integration, Aplication and Teaching - Descubrimiento, Integración, Aplicación y Enseñanza) de Boyer (1990), ampliamente utilizado en los modelos de desarrollo profesional en la era digital (Constantino y Raffaghelli, 2016) podemos observar dos tipos de los desarrollos relacionados con la alfabetización de datos.

En primer lugar la investigación, que para el modelo de Boyer equivale a las actividades de descubrimiento, integración y aplicación, y por ello devenir un académico en un contexto digital impulsado por datos implica formas de activismo que tienen en cuenta las preocupaciones de justicia social y ética al realizar investigaciones. El marco de la Investigación e Innovación Responsable impulsado por la Comisión Europea a través de su programa marco de investigación Horizon 2020 (Responsible Research y Innovation, o RRI, Owen, Macnaghten y Stilgoe, 2012), que incorpora el concepto de datos abiertos (Open Data), es una política de la Unión Europea (UE) que aborda inquietudes prácticas sobre cómo se lleva a cabo la investigación en contextos abiertos y participativos, donde la investigación financiada con fondos públicos debe dejar sus datos como legado del cuál la población puede apropiarse. Además, la literatura emergente sobre el profesionalismo académico en los entornos digitales, abiertos y en red (Goodfellow, 2014; Juliana Elisa Raffaghelli, 2017b; Veletsianos y Stewart, 2016; Veletsianos, 2013; Veletsianos y Kimmons, 2012) requiere una actualización en el sentido de comprender que una dimensión del uso de entornos y herramientas digitales implicará por fuerza una visibilidad o uso de datos por parte inclusive de terceros, que debe ser reconocida (Hey, 2009).

Mucho más complejo aún es el segundo relacionado con la enseñanza (Teaching), que para Boyer se convierte en una única 
dimensión en su modelo DIAT. En este ámbito, por un lado los datos pueden usarse en el proceso educativo y existen numerosos marcos de competencia relacionados con las habilidades técnicas en gestión de datos a promover entre los alumnos (Maybee y Zilinski, 2015). Sin embargo, desde hace unos 20 años toda la investigación en materia de gestión escolar ha mostrado una perspectiva sobre el uso de datos para informar de las prácticas de planificación educativa y del mejoramiento de la práctica pedagógica (Mandinach, 2012; Mandinach y Gummer, 2016) que han sido ampliamente relacionadas con el modelo de educación basada en evidencias (Evidence-based Education, Slavin, 2002). En la educación superior, este debate podría relacionarse con las analíticas de aprendizaje y sus múltiples desarrollos, donde la alfabetización en datos del profesorado y de los estudiantes equivale a comprender los sistemas de trazado de datos, los algoritmos utilizados para generar cuadros de diagnóstico y de predicción de comportamientos en un sistema de aprendizaje, y la lectura de las interfaces gráficas (dashboards) con analíticas visuales. En este sentido, la alfabetización en datos se relacionaría con una especie de alfabetización pedagógica, es decir, a la traducción de los datos recabados en constructos pedagógicos a partir (Raffaghelli, 2018a; Wasson, Hansen y Netteland, 2016).

Haciendo referencia al marco de profesionalismo académico de Boyer que reclamaba la investigación didáctica (modelos SOTL, Scholarship of Teaching and Learning, relacionado con la subdimensión DIAT de la enseñanza de Boyer antes citada) el uso de datos podría llevar a formas más significativas de analizar, evaluar y compartir prácticas pedagógicas efectivas. Inclusive, se podría proyectar una visión estratégica de la educación como ciencia abierta (Tim Zee, 2018), mientras que los conjuntos de datos de la investigación basada en el diseño educativo podrían ser comentados críticamente y compartidos en la amplia comunidad educativa (Persico y Pozzi, 2015). En este caso, también estamos al comienzo de las prácticas y del compromiso que deben explorarse más a fondo (Raffaghelli y Manca, 2019). Sin embargo, estos modelos podrían parecer demasiado técnicos o inclusive naïf en sus asunciones de eficacia pedagógica objetivista, sin considerar 
las realidades socio-culturales complejas y cambiantes en las que se plantean las intervenciones. Por ejemplo Pangrazio yy Selwyn (2018) han reclamado la necesidad de implementar formas de alfabetización en datos personales que permitan al usuario tener un mayor control no solo en la forma en que son trazados sus datos, sino también en la forma en que interactúa con un sistema para producir o disminuir la producción de datos en un sistema digital.

Más allá de una visión distópica de los datos en las prácticas académicas que enfatiza el control, la vigilancia y la falta de preocupaciones éticas con respecto a la recopilación de datos (Williamson, 2018), los procesos de toma de conciencia y de negociación de sentido basados en las infraestructuras existentes podrían generar prácticas que implicarían el aprendizaje profesional continuo y el desarrollo organizacional hacia la apropiación del fenómeno de datificación. En este sentido se tendría que considerar de manera muy especial, , que la mayor parte de las universidades a nivel global (y particularmente en el Sud global) no poseen almacenes de datos (data warehouse) ni capacidad de procesamiento para implementar los complejos sistemas de analíticas predictivas y de visualización que tanto han preocupado a la comunidad internacional (véase por ejemplo el caso de la crítica a Pearson Education en Williamson, 2018); por lo tanto la «datificación» tiene que ser leída desde la perdsona participante, como invitarían a hacerlo Pangrazio y Selwyn (2018), desde el minimalismo de los hábitos de almacenamiento, de compartición y elaboración de datos (p. e. tener clara la diferencia entre usar un LMS local y algunas redes sociales como Facebook o Instagram, actualmente tan promovidas).

En fin, el uso final de los datos generados en los procesos de investigación, la enseñanza y el aprendizaje, al ser discutido y consensuado, a través del diálogo entre los académicos y la sociedad además de muy fructífero, podría convertirse en un trampolín para aunar esfuerzos comunes en relación a escenarios de justicia social de cara al avance tecnológico de la datificación. 


\section{Alfabetización en datos: la configuración de un nuevo escenario formativo}

Pero..., ¿Qué es la alfabetización de datos? En el informe canadiense de 2015 promovido por el Consejo Nacional de Ciencias Humanísticas Sociales sobre la alfabetización de datos, se presenta como la capacidad de recopilar, gestionar, evaluar y utilizar los datos en contextos de aplicación de manera crítica (Ridsdale et al., 2015). Esta capacidad se convierte en competencia cuando se puede poner en contexto tanto a nivel académico (manipulación con fines de comunicación académica), dentro de su propio sector disciplinario; como a nivel profesional, cuando los datos se utilizan para informar procesos y decisiones (nivel más esencial) o también para generar productos y servicios (nivel avanzado e innovación). Las diferentes definiciones existentes en el ámbito académico y de investigación coinciden en los siguientes elementos clave: extracción, gestión y procesamiento, y enfoque ético crítico.

Tradicionalmente, el constructo ha permanecido implícito en el concepto más amplio de la alfabetización informacional (information literacy), y creció dentro de él hasta el momento de obtener un estado propio. Por ejemplo, los modelos de alfabetización informacional buscan cultivar: a) habilidades de investigación y análisis de información general; b) uso crítico de la información dentro de entornos diversificados; c) uso crítico y ético de la información para transformar el mundo (Lupton y Bruce, 2010). Más allá de estas amplias categorías, los enfoques de alfabetización de datos se centraron inicialmente en actividades que se realizarían en contextos formales, en particular relacionados con la enseñanza de las ciencias y las matemáticas (Stephenson y Schifter Caravello, 2007). Sin embargo, el debate en el contexto de la alfabetización informacional ha sentado las bases para sugerir enfoques más ricos, más allá del aprendizaje formal, considerando no solo aspectos cognitivos sino también éticos y creativos (Carlson, Fosmire, Miller, y Nelson, 2011). Coincidimos con la revisión de la literatura realizada por Maybee y Zilinski (2015), basada en el 
análisis de 8 marcos de alfabetización de datos, en los que identificaron los siguientes elementos:

1. Conciencia: entender qué son los datos y cuál es su papel para la sociedad.

2. Acceso: comprender cómo identificar, localizar y usar correctamente los conjuntos de datos y las bases de datos.

3. Participación: evaluar, analizar, organizar e interpretar los datos existentes y tomar decisiones basadas en ellos.

4. Administración: planificar y administrar datos, incluidos la organización y el análisis, los protocolos de seguridad para su almacenamiento, intercambio y la documentación asociada.

5. Comunicación: sintetizar, crear visualizaciones y representación de datos.

6. Uso ético: identificar fuentes de datos diversificadas, en particular relacionadas con la actividad humana y social, considerando los riesgos de administrar dichos datos, comprendiendo las cuestiones implícitas en su uso.

7. Conservación: conocer las formas de seguridad a corto y largo plazo, en relación al almacenamiento, uso y reutilización de datos.

Los enfoques no han sido formulados sólo desde el ámbito de la investigación: a medida que el fenómeno de la «datificación» crecía, sino que se han ido agregando componentes y enfoques. Por ejemplo, del movimiento de datos abiertos, han surgido interpretaciones sobre las habilidades necesarias para operar en el sector. Un ejemplo es el marco de Open Data Skills, generado por el Open Data Institute ${ }^{2}$ (ODI), una organización que promueve la capacitación avanzada en las áreas de la ciencia de datos y las habilidades profesionales básicas, que categoriza a los usuario según los siguientes niveles de trabajo con datos: 
1. Explorador: que tiene una comprensión básica de los datos. En este nivel puede definir los datos con los que trabajar, indicar ejemplos o estudios de casos y explicar cómo se pueden usar los datos para crear un cambio.

2. Profesional; que realiza operaciones básicas en un conjunto de datos abiertos. Puede navegar por los datos y conoce las herramientas y técnicas necesarias para administrar y publicar un conjunto de datos abiertos.

3. Estratega: que integra datos abiertos en una estrategia o administra un proyecto de datos abiertos. Conoce las técnicas de planificación y gestión para llevar a cabo una iniciativa de datos abiertos y comprende los desafíos inherentes a este proceso.

4. Pionero: que posee habilidades y conocimientos que les permiten resolver desafíos en su sector. Puede indicar estudios de casos específicos por sector, identificar tendencias futuras en el sector y comprender cómo utilizar los datos que mejor se ajustan a los desafíos de producción específicos de su sector.

En una segunda fase, el ODI ha producido un marco interactivo donde se va más allá de los niveles de competencia para enfocar las áreas y los contenidos de formación en ciencia de datos en contextos profesionales. En estos casos, han apuntado a formar competencias básicas de introducción al uso de datos, su publicación, gestión, producción o desarrollo basados en macrodatos o datos abiertos, y análisis y liderazgo de $s$ modelos de negocios. Sin embargo, para la ODI, dichas competencias podrían ser activos de una organización, no necesariamente de un solo trabajador, por lo tanto, su oferta de capacitación y el marco relacionado es parte de un plan de desarrollo organizacional como una "organización basada en la ciencia de datos".

Como se puede inferir, este marco se refiere a las habilidades profesionales, que pueden crear un escenario prospectivo para el desarrollo de la alfabetización en datos desde las primeras etapas de la formación, mientras que las iniciativas de formación continua se enfocan más como objetivo final. Sin embargo, parece necesario 
proponer bases de trabajo para la educación formal y, por lo tanto, para la formación de profesores y formadores.

En este ejercicio, podríamos introducir el debate europeo sobre el marco de Competencia Digital, DigComp 2.1. (Carretero, Vuorikari, y Punie, 2017). El concepto, ya presente en el primer marco de las denominadas "Competencias Clave para el Aprendizaje a lo largo de toda la vida" de 2006 (European Commission, 2007), exige mayor atención en los elementos ofrecidos por los contextos y herramientas de la era digital, y por lo tanto invita a pensar en una serie de conocimiento y habilidades generalizadas para todas las acciones de la vida diaria y profesional en contextos digitales. El primer marco DigComp nació en el contexto de las acciones de la Agencia Digital Europea $2020^{3}$ y respondió a la gran necesidad de calificar y generar entornos digitales calificados para todos los ciudadanos europeos de todas las edades. Se publicó una nueva versión en 2016 (2.0); sin embargo, el cambio que más nos interesa es el de la última actualización de 2017 (2.1) en la que, bajo el encabezado "Alfabetización de la información", se agrega el elemento "Alfabetización de datos". El nuevo marco ofrece no solo la definición de competencias, sino que aporta una serie de escenarios en los que se deben aplicar estas competencias y que demuestra su logro. Por lo tanto, dicha estructura debería permitir la evaluación de las competencias digitales en sus 8 componentes, incluida la citada alfabetización en datos. Sin embargo, la lectura cuidadosa de todo el modelo y de este subcomponente, así como de las situaciones consideradas para su evaluación, parecen insuficientes para abarcar toda la riqueza de la alfabetización de datos en los componentes que hemos descrito anteriormente. De hecho, en DigComp 2.1. se habla de alfabetización en datos en términos de:

1.1. Explorar, buscar y filtrar datos, información y contenido digital (en la versión DigComp 1.0: navegar, buscar y filtrar información)

1.2. Evaluar datos, información y contenido digital (en la versión DigComp 1.0: evaluar información) 


\subsection{Gestionar datos, información y contenido digital} (en la versión DigComp 1.0: Almacenar y recuperar información).

Pero en estos tres subcomponentes, no aparece ninguno de los aspectos éticos, creativos y constructivos de la alfabetización de datos.

De hecho DigComp 2.1. ofrece áreas de análisis sucesivas que podrían ofrecer espacio a las formas más creativas de alfabetización de datos, este componente no se identifica adecuadamente y se pierde entre las muchas otras actividades que deben considerarse en las dimensiones posteriores. Al alinear las dimensiones de DigComp con los elementos de alfabetización de datos ya analizados, encontramos que; a) la dimensión de comunicación y colaboración tiene un ejemplo asociado a la alfabetización de datos en los procesos de documentación y narración con datos, es decir, la narración de datos; b) la dimensión de creación de contenidos digitales, lo tiene asociado en visualizaciones de datos; c) la dimensión de seguridad, en la anonimización de datos; y finalmente, d) la dimensión de resolución de problemas en las extracciones complejas y elaboraciones estadísticas.

Estos elementos sientan las bases para pensar en un marco de competencias que apoyan la alfabetización de datos que, aunque converge con DigComp, debe encontrar su espacio, respondiendo a las necesidades de capacitación que se discutieron en los párrafos iniciales.

Más aún, observamos que la introducción del marco DigCompEdu, de competencia digital de educadores asociado a DigComp, puede ser discutido a la hora de pensar la alfabetización en datos de los mismos educadores, y en particular del profesorado universitario. 


\section{Hacia la conceptualización de un marco para la alfabetización en datos en el desarrollo profesional del profesorado}

Como hemos indicado más arriba, las prácticas de datos más constructivas y críticas dentro de la academia requieren un concepto complejo de alfabetización de datos del profesorado y de los estudiantes de educación superior. La alfabetización de datos también debería integrarse al concepto general de competencia profesional de profesorado o competencia docente, que abarca, entre otras, las prácticas profesionales, las condiciones de trabajo y las identidades de los académicos en el contexto de educación superior digitalizada (Raffaghelli, 2018a). Sin embargo todavía hay escasez de investigación conceptual y empírica sobre desarrollo del profesorado universitario en alfabetización en datos («data literacy») Esto podría deberse al hecho de que los problemas relacionados con los datos están diversificados, y posicionados en los dos campos complejos de la investigación y de la enseñanza, como se comentó anteriormente. En relación con la investigación, los datos están relacionados con los discursos de la ciencia abierta y los datos abiertos en la investigación, y en relación con la enseñanza, los datos provienen de las prácticas pedagógicas y de las actividades e interacciones de los profesores y los alumnos en el aprendizaje en línea y semipresencial o mixto. Estos son dos universos de investigación poseen enfoques y constructos diferentes., pero las prácticas basadas en datos implican formas de conocimiento que son potencialmente transversales en un contexto de modernización de la educación superior. La pregunta de investigación que ha orientado esta exploración conceptual ha sido: ¿Cómo debe diseñarse y desarrollarse el desarrollo profesional del personal académico para introducir un uso transformativo y crítico de los datos digitalizados y asegurar un impacto positivo en la alfabetización en datos (Data Literacy - DL) como un componente esencial en la modernización de la educación superior?

Esta pregunta de investigación es muy amplia y plantea un trabajo de investigación que requiere no sólo la identificación de 
componentes a formar, sino también la experimentación formativa y de procesos de desarrollo profesional con sus implicaciones para el desarrollo organizacional y el impacto en los jóvenes universitarios. Sin embargo, la definición de constructos y de marcos de competencia resulta un ejercicio fundamental para la sucesiva implementación de actividades en campo.

El constructo principal subyacente es el de desarrollo de competencia digital en el profesorado, vinculado a las prácticas abiertas, en red y digitales llevadas a cabo por académicos en sus actividades profesionales. Este concepto, ha sido tratado de varias maneras y con distintos enfoques pero recientemente se ha caracterizado como "Digital Scholarship», que podemos traducir como «trabajo académico digital» (Li, Greenhow, y Askari, 2016; Manca y Ranieri, 2017; Pearce, Weller, Scanlon, y Kinsley, 2010; Raffaghelli, 2017b; Stewart, 2015). Como ha sido puesto en evidencia en la revisión de la literatura, las discusiones sobre qué aspectos deben ser considerados como parte de la «Digital Scholarship» están bastante diversificados entre didáctica e investigación, lo que complejiza el escenario ( Raffaghelli, Cucchiara, Manganello y Persico, 2016; Raffaghelli, 2017b). Resulta claro que mientras existe en el profesorado universitario un área de competencia ligada a la investigación, que muy frecuentemente puede ser tratada con los mismos parámetros de "data literacy» aplicados a la ciencia de datos en su cruce con los criterios de ciencia abierta («Open Science»), la alfabetización de datos para la didáctica tiene coincidencias con otros ámbitos de la educación, si bien posee sus aspectos característicos.

Queriendo ocuparnos aquí de una caracterización conceptual y operativa de la alfabetización en datos, debemos recurrir a los marcos de competencia digital, y en este sentido, hacer referencia a un marco de competencia digital de educadores que ya se encuentra desarrollado, el marco de la Unión Europea (UE) por medio de DigCompEdu (Redecker y Punie, 2017), el excurso de análisis del problema (la necesidad de un marco de alfabetización en datos) y de la revisión de la literatura, posibilita realizar una adaptación conceptual de dicho marco. 
A pesar de que la aplicación de este marco a la profesionalidad de los académicos puede ser controvertida, ya que se desarrolló para abordar el profesionalismo de los educadores, argumentamos que la relevancia en los objetivos institucionales de la educación superior de la tarea didáctica, hace que los académicos sean una categoría específica de educadores. Sin embargo, nuestro marco no excluye el hecho de que los académicos son a veces los productores del conocimiento que enseñan, en calidad de investigadores. Por ello, hemos considerado áreas de competencia donde los nichos de la investigación y la enseñanza en el mundo académico pueden conectarse y la investigación puede incluirse parcialmente en el marco. Este es el caso de los datos de investigación utilizados como contenido educativo (Atenas et al., 2015); en este marco, el objetivo del trabajo de conceptualización de un marco sería el de ofrecer un instrumento para encuadrar, analizar o promover procesos de alfabetización en datos en la enseñanza y el aprendizaje universitarios en un ciclo complejo que considera los datos y la «datificación» bien como recurso educativo, bien como elemento transversal de la práctica profesional y personal en la vida universitaria.

La Tabla 1 muestra las dimensiones de competencia relacionadas con un estado final y elevado de alfabetización en datos. Dicha tabla ha tomado en consideración las mismas dimensiones de competencia de DigCompEdu y las ha adaptado en relación a la revisión de literatura de modelos de alfabetización en datos anteriormente presentada. La Tabla 2, en cambio, ofrece un panorama dinámico de evolución de dicha competencia, siempre asociada al marco DigCompEdu, que primero presenta la competencia digital y sus dimensiones, y luego ofrece una gradación de desarrollo de la misma como referencia para el diagnóstico o autodiagnóstico. 
Tabla 1. Marco de referencia para el desarrollo de la alfabetización en datos para el profesorado universitario.

\begin{tabular}{|c|c|c|}
\hline $\begin{array}{l}\text { Dimensión de la } \\
\text { competencia }\end{array}$ & Descriptor & Detalle descriptor \\
\hline \multirow{5}{*}{$\begin{array}{l}\text { CONTEXTO DE } \\
\text { ACTUACIÓN } \\
\text { PROFESIONAL }\end{array}$} & $\begin{array}{l}\text { Ciencia de datos } \\
\text { en la investigación }\end{array}$ & $\begin{array}{l}\text { A lo largo del ciclo completo de la información científica, debe } \\
\text { participar activamente en la ciencia abierta y en las prácticas } \\
\text { avanzadas de datos que son eventualmente transferibles a la } \\
\text { enseñanza. }\end{array}$ \\
\hline & $\begin{array}{l}\text { Comunicación } \\
\text { organizacional }\end{array}$ & $\begin{array}{l}\text { Utilizar los datos disponibles para mejorar la comunicación } \\
\text { organizativa con colegas, estudiantes y terceros. Contribuir al } \\
\text { desarrollo y la mejora de las estrategias y políticas de comunicación } \\
\text { organizacional impulsadas por datos (aprendizaje y análisis } \\
\text { académico, informes estadísticos, datos de redes sociales, etc.). }\end{array}$ \\
\hline & $\begin{array}{l}\text { Colaboración } \\
\text { profesional }\end{array}$ & $\begin{array}{c}\text { Utilizar enfoques de datos abiertos en actividades científicas y } \\
\text { educativas para interactuar con otros académicos, compartir e } \\
\text { intercambiar conocimientos y experiencias y prácticas pedagógicas } \\
\text { innovadoras en colaboración. }\end{array}$ \\
\hline & Práctica reflexiva & $\begin{array}{c}\text { Evaluar críticamente y desarrollar activamente las propias prácticas } \\
\text { basadas en datos dentro de la enseñanza. } \\
\text { Reflexionar sobre las conexiones entre el avance de la propia } \\
\text { disciplina de investigación y los procedimientos de datos integrados } \\
\text { en la enseñanza. }\end{array}$ \\
\hline & $\begin{array}{l}\text { Desarrollo } \\
\text { profesional } \\
\text { continuo. }\end{array}$ & $\begin{array}{l}\text { Cultivar ecologías de aprendizaje apoyando la alfabetización de } \\
\text { datos en la práctica académica. }\end{array}$ \\
\hline \multirow{3}{*}{$\begin{array}{l}\text { LOS DATOS } \\
\text { COMO } \\
\text { RECURSOS } \\
\text { PARA EL } \\
\text { APRENDIZAJE }\end{array}$} & $\begin{array}{l}\text { Selección de datos } \\
\text { como recurso para } \\
\text { el aprendizaje. }\end{array}$ & $\begin{array}{c}\text { Identificar, evaluar y seleccionar datos o enfoques de datos como } \\
\text { recursos para la enseñanza y el aprendizaje. } \\
\text { Considerar objetivos de aprendizaje específicos, contextos, } \\
\text { enfoques pedagógicos y grupos de estudiantes al diseñar enfoques } \\
\text { didácticos con uso de datos. }\end{array}$ \\
\hline & $\begin{array}{l}\text { Generación, } \\
\text { recolección, } \\
\text { extracción de } \\
\text { datos como } \\
\text { recurso para el } \\
\text { aprendizaje. }\end{array}$ & $\begin{array}{l}\text { Planificar la extracción, modificación y elaboración de datos } \\
\text { abiertos (Open Data) como parte de tareas de aprendizaje } \\
\text { auténticas, basadas en problemas y orientadas a proyectos }\end{array}$ \\
\hline & $\begin{array}{l}\text { Gestionar, } \\
\text { proteger y } \\
\text { compartir datos. }\end{array}$ & $\begin{array}{l}\text { Organizar los datos como contenido educativo y ponerlos a } \\
\text { disposición de los alumnos y, eventualmente, de otras partes } \\
\text { interesadas. Disponer los datos seleccionados para la enseñanza, } \\
\text { protegiendo con eficacia los datos sensibles; respetando y } \\
\text { aplicando correctamente las reglas de privacidad y derechos de } \\
\text { autor para abrir datos; generando formas de comprensión del uso } \\
\text { de licencias abiertas incluida su atribución adecuada. }\end{array}$ \\
\hline \multirow{3}{*}{$\begin{array}{l}\text { PROCESOS DE } \\
\text { ENSEÑANZAY } \\
\text { APRENDIZAJE }\end{array}$} & Enseñanza & $\begin{array}{c}\text { Planificar e implementar actividades y visualizaciones basadas en } \\
\text { datos en el proceso de enseñanza y aprendizaje, a fin de mejorar la } \\
\text { eficacia de las intervenciones de enseñanza. }\end{array}$ \\
\hline & Guía/Tutorización & $\begin{array}{l}\text { Integrar los datos (presentes en registros de los estudiantes, } \\
\text { evaluaciones, evaluaciones de cursos, procesos de monitoreo, } \\
\text { paneles) para ofrecer orientación y asistencia oportunas y } \\
\text { específicas. Experimentar y desarrollar nuevos formularios y } \\
\text { formatos para ofrecer orientación y soporte basados en datos. }\end{array}$ \\
\hline & $\begin{array}{l}\text { Aprendizaje } \\
\text { colaborativo }\end{array}$ & $\begin{array}{c}\text { Utilizar enfoques basados en datos para fomentar y mejorar la } \\
\text { colaboración de los alumnos. Para permitir que los alumnos utilicen } \\
\text { sus propios datos como parte de tareas colaborativas y como un } \\
\text { medio para mejorar la comunicación y la colaboración y la creación } \\
\text { de conocimiento colaborativo. }\end{array}$ \\
\hline
\end{tabular}




\begin{tabular}{|c|c|c|}
\hline $\begin{array}{l}\text { Dimensión de la } \\
\text { competencia }\end{array}$ & Descriptor & Detalle descriptor \\
\hline $\begin{array}{l}\text { PROCESOS DE } \\
\text { ENSEÑANZAY } \\
\text { APRENDIZAJE }\end{array}$ & $\begin{array}{l}\text { Aprendizaje } \\
\text { autorregulado }\end{array}$ & $\begin{array}{c}\text { Utilizar enfoques basados en datos (a partir de registros de los } \\
\text { estudiantes, evaluaciones, evaluaciones de cursos, procesos } \\
\text { de monitoreo, paneles de control) para apoyar el aprendizaje } \\
\text { autorregulado de los estudiantes, es decir, para permitir que } \\
\text { los estudiantes planifiquen, monitoreen y reflexionen sobre su } \\
\text { propio aprendizaje, que busquen, comprendan, compartan ideas, } \\
\text { y encuentren soluciones creativas sobre la evidencia del propio } \\
\text { progreso. }\end{array}$ \\
\hline \multirow[b]{3}{*}{ EVALUACIÓN } & $\begin{array}{l}\text { Estrategias de } \\
\text { evaluación }\end{array}$ & $\begin{array}{c}\text { Integrar los datos producidos a lo largo del proceso de aprendizaje } \\
\text { con fines de evaluación formativa y sumativa. }\end{array}$ \\
\hline & $\begin{array}{l}\text { Análisis de la } \\
\text { evidencia }\end{array}$ & $\begin{array}{l}\text { Generar, seleccionar, analizar críticamente e interpretar los datos } \\
\text { como evidencia de la actividad, el rendimiento y el progreso del } \\
\text { alumno, a fin de apoyar procesos de diseño didáctico. }\end{array}$ \\
\hline & $\begin{array}{l}\text { Retroalimentación } \\
\text { y planificación }\end{array}$ & $\begin{array}{l}\text { Utilizar los datos producidos a lo largo del proceso de aprendizaje } \\
\text { para proporcionar retroalimentación dirigida y oportuna a los } \\
\text { alumnos. Adaptar las estrategias de enseñanza y proporcionar } \\
\text { un apoyo específico basado en la evidencia generada por las } \\
\text { tecnologías digitales utilizadas. Permitir que los estudiantes y } \\
\text { otras partes interesadas entiendan los datos como una forma de } \\
\text { evidencia proporcionada por tecnologías digitales. }\end{array}$ \\
\hline \multirow{3}{*}{$\begin{array}{l}\text { EMPODERAMIENTO } \\
\text { DELOS } \\
\text { ESTUDIANTES }\end{array}$} & $\begin{array}{l}\text { Accesibilidad e } \\
\text { inclusión }\end{array}$ & $\begin{array}{c}\text { Garantizar el acceso a los datos generados y utilizados durante } \\
\text { las actividades de aprendizaje, de todos los alumnos, incluidos } \\
\text { aquellos con necesidades especiales. Considerar y responder } \\
\text { a las expectativas, habilidades, usos y conceptos erróneos de } \\
\text { los alumnos sobre los datos manipulados durante procesos de } \\
\text { enseñanza y aprendizaje. }\end{array}$ \\
\hline & $\begin{array}{l}\text { Diferenciación y } \\
\text { personalización. }\end{array}$ & $\begin{array}{l}\text { Usar las tecnologías digitales para abordar las diversas } \\
\text { necesidades de aprendizaje de los alumnos, permitiendo a los } \\
\text { alumnos avanzar a diferentes niveles y velocidades y seguir } \\
\text { caminos y objetivos de aprendizaje individuales. }\end{array}$ \\
\hline & $\begin{array}{l}\text { Participación } \\
\text { active de los } \\
\text { estudiantes. }\end{array}$ & $\begin{array}{c}\text { Usar tecnologías digitales (como la narración de datos y la } \\
\text { generación de infografías) para fomentar el compromiso creativo } \\
\text { y crítico de los alumnos con los datos como contenido de } \\
\text { aprendizaje. Abrir el aprendizaje a nuevos contextos de uso de } \\
\text { datos en el mundo real, involucrando a los propios alumnos en } \\
\text { actividades prácticas, investigación científica o resolución de } \\
\text { problemas complejos. }\end{array}$ \\
\hline \multirow{4}{*}{$\begin{array}{l}\text { FACILITAR LA } \\
\text { ALFABETIZACIÓN } \\
\text { DE DATOS } \\
\text { AUTÓNOMA }\end{array}$} & $\begin{array}{l}\text { Capacidad técnica } \\
\text { de los alumnos } \\
\text { para manejar los } \\
\text { datos. }\end{array}$ & $\begin{array}{c}\text { Incorporar actividades de aprendizaje, tareas y evaluaciones que } \\
\text { requieran que los estudiantes se interroguen sobre qué nuevas } \\
\text { fuentes de datos consultar, extraer o crear; ayudar a los estudiantes } \\
\text { a encontrar y extraer datos sin procesar en entornos digitales; } \\
\text { apoyar a los alumnos en la organización, procesamiento, análisis } \\
\text { e interpretación de datos para comparar y evaluar críticamente la } \\
\text { credibilidad y confiabilidad de los datos disponibles, en relación a } \\
\text { las fuentes de extracción. }\end{array}$ \\
\hline & $\begin{array}{l}\text { Uso de los datos } \\
\text { de los alumnos en } \\
\text { la comunicación y } \\
\text { colaboración. }\end{array}$ & $\begin{array}{l}\text { Incorporar actividades de aprendizaje, tareas y evaluaciones que } \\
\text { requieran que los estudiantes usen herramientas digitales de } \\
\text { manera efectiva y responsable para compartir, comentar, elaborar y } \\
\text { presentar datos. }\end{array}$ \\
\hline & $\begin{array}{l}\text { Habilidades de } \\
\text { los alumnos en la } \\
\text { narración basada } \\
\quad \text { en datos. }\end{array}$ & $\begin{array}{l}\text { Incorporar actividades de aprendizaje, tareas y evaluaciones que } \\
\text { promuevan en los alumnos la generación de visualizaciones, } \\
\text { representaciones e historias utilizando datos. Enseñar a los } \\
\text { alumnos cómo se aplican los derechos de autor y las licencias } \\
\text { existentes a los datos como contenido digital. }\end{array}$ \\
\hline & $\begin{array}{l}\text { Uso responsable } \\
\text { de los datos } \\
\text { por parte de los } \\
\text { estudiantes. }\end{array}$ & $\begin{array}{l}\text { Permitir a los alumnos reconocer y gestionar los riesgos de usar } \\
\text { datos personales, sociales y generalmente abiertos de forma } \\
\text { segura y responsable. }\end{array}$ \\
\hline
\end{tabular}




\begin{tabular}{|l|c|c|}
\hline $\begin{array}{l}\text { Dimensión de la } \\
\text { competencia }\end{array}$ & Descriptor & Detalle descriptor \\
\hline $\begin{array}{l}\text { FACILITAR LA } \\
\text { ALFABETIZACIÓN } \\
\begin{array}{l}\text { DE DATOS } \\
\text { AUTÓNOMA }\end{array}\end{array}$ & $\begin{array}{c}\text { Uso de los datos } \\
\text { de los alumnos en } \\
\text { la resolución de } \\
\text { problemas. }\end{array}$ & $\begin{array}{c}\text { Incorporar actividades de aprendizaje, tareas y evaluaciones que } \\
\text { ayuden a los alumnos a identificar y resolver problemas técnicos y } \\
\text { extracción de datos, elaboración y presentación. }\end{array}$ \\
\hline
\end{tabular}

Fuente. Elaboración propia.

Tabla 2. Niveles dinámicos de desarrollo en el uso de datos .

\begin{tabular}{|c|c|c|c|c|c|c|}
\hline 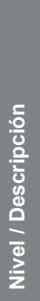 & 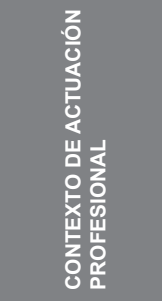 & 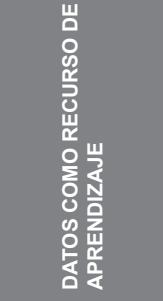 & 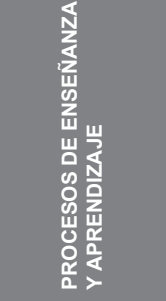 & 吕 & 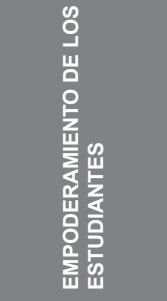 & 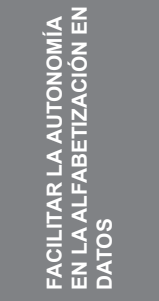 \\
\hline $\begin{array}{l}\frac{0}{0} \\
\frac{0}{0} \\
\frac{0}{0} \\
\text { J }\end{array}$ & $\begin{array}{l}\text { Innovar hacia } \\
\text { una perspectiva } \\
\text { crítica de } \\
\text { las prácticas } \\
\text { profesionales } \\
\text { basadas en } \\
\text { datos. }\end{array}$ & $\begin{array}{c}\text { Promover } \\
\text { formas } \\
\text { innovadoras de } \\
\text { explorar y usar } \\
\text { datos. }\end{array}$ & $\begin{array}{l}\text { Innovar en } \\
\text { formas de } \\
\text { entender y usar } \\
\text { datos en la } \\
\text { enseñanza y el } \\
\text { aprendizaje. }\end{array}$ & $\begin{array}{l}\text { Innovar en } \\
\text { formas de } \\
\text { entender } \\
\text { y utilizar la } \\
\text { evaluación de } \\
\text { datos }\end{array}$ & $\begin{array}{l}\text { Participación } \\
\text { innovadora } \\
\text { del alumno } \\
\text { en prácticas } \\
\text { basadas en } \\
\text { datos }\end{array}$ & $\begin{array}{l}\text { Uso de formatos } \\
\text { innovadores } \\
\text { para fomentar } \\
\text { el conocimiento } \\
\text { personal, } \\
\text { profesional y de } \\
\text { datos sociales } \\
\text { de los alumnos. }\end{array}$ \\
\hline $\begin{array}{l}\frac{1}{0} \\
\frac{0}{J} \\
\overline{0}\end{array}$ & $\begin{array}{l}\text { Discutir y } \\
\text { renovar } \\
\text { prácticas } \\
\text { profesionales } \\
\text { basadas en } \\
\text { datos. }\end{array}$ & $\begin{array}{l}\text { Uso integral } \\
\text { de estrategias } \\
\text { y recursos } \\
\text { avanzados para } \\
\text { explorar y usar } \\
\text { datos }\end{array}$ & $\begin{array}{c}\text { Renovar } \\
\text { estratégica y } \\
\text { deliberadamente } \\
\text { la práctica } \\
\text { docente } \\
\text { mediante la } \\
\text { comprensión y } \\
\text { el uso de datos. }\end{array}$ & $\begin{array}{l}\text { Reflexionar } \\
\text { críticamente } \\
\text { sobre las formas } \\
\text { de entender } \\
\text { y utilizar los } \\
\text { datos en la } \\
\text { evaluación. }\end{array}$ & $\begin{array}{c}\text { Capacitar } \\
\text { holísticamente a } \\
\text { los estudiantes } \\
\text { en prácticas } \\
\text { basadas en } \\
\text { datos }\end{array}$ & $\begin{array}{c}\text { Fomento } \\
\text { integral y } \\
\text { crítico de la } \\
\text { alfabetización } \\
\text { de datos de los } \\
\text { alumnos. }\end{array}$ \\
\hline 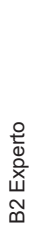 & $\begin{array}{l}\text { Mejora de } \\
\text { datos dentro } \\
\text { de las prácticas } \\
\text { profesionales. }\end{array}$ & $\begin{array}{c}\text { Uso de } \\
\text { estrategias } \\
\text { y recursos } \\
\text { interactivos para } \\
\text { explorar y usar } \\
\text { datos. }\end{array}$ & $\begin{array}{l}\text { Mejora de las } \\
\text { actividades de } \\
\text { enseñanza y } \\
\text { aprendizaje a } \\
\text { través de los } \\
\text { datos. }\end{array}$ & $\begin{array}{l}\text { Uso estratégico } \\
\text { y efectivo de } \\
\text { los datos en la } \\
\text { evaluación. }\end{array}$ & $\begin{array}{l}\text { Uso estratégico } \\
\text { de una gama } \\
\text { de herramientas } \\
\text { para capacitar a } \\
\text { los estudiantes } \\
\text { en prácticas } \\
\text { basadas en } \\
\text { datos }\end{array}$ & $\begin{array}{c}\text { Fomento } \\
\text { estratégico de } \\
\text { la alfabetización } \\
\text { de datos de los } \\
\text { alumnos. }\end{array}$ \\
\hline 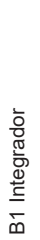 & $\begin{array}{l}\text { Expansión } \\
\text { de la práctica } \\
\text { profesional } \\
\text { mediante el uso } \\
\text { de datos. }\end{array}$ & $\begin{array}{l}\text { Comprensión de } \\
\text { posibles usos } \\
\text { de los datos } \\
\text { como recurso } \\
\text { de aprendizaje. }\end{array}$ & $\begin{array}{l}\text { Integración } \\
\text { significativa } \\
\text { de datos en } \\
\text { la práctica } \\
\text { docente. }\end{array}$ & $\begin{array}{l}\text { Mejora de la } \\
\text { evaluación } \\
\text { tradicional } \\
\text { sobre la base } \\
\text { de los datos } \\
\text { disponibles }\end{array}$ & $\begin{array}{l}\text { Comprensión } \\
\text { de la posibilidad } \\
\text { de empoderar a } \\
\text { los estudiantes } \\
\text { a través de } \\
\text { prácticas } \\
\text { basadas en } \\
\text { datos }\end{array}$ & $\begin{array}{l}\text { Implementación } \\
\text { de actividades } \\
\text { eventuales para } \\
\text { fomentar la } \\
\text { alfabetización } \\
\text { de datos de los } \\
\text { estudiantes. }\end{array}$ \\
\hline
\end{tabular}




\begin{tabular}{|c|c|c|c|c|c|c|}
\hline 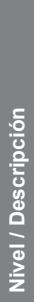 & 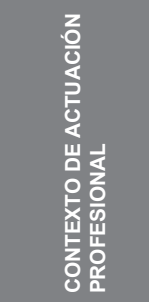 & 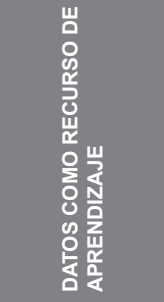 & 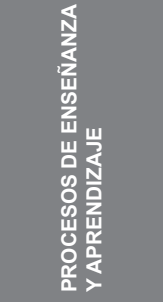 & 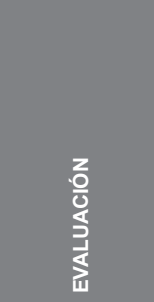 & 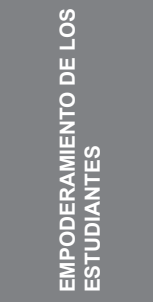 & 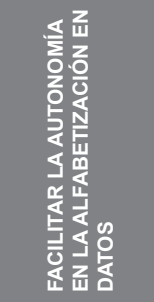 \\
\hline 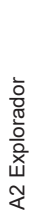 & $\begin{array}{l}\text { Exploración de } \\
\text { otras prácticas } \\
\text { profesionales } \\
\text { basadas en } \\
\text { datos }\end{array}$ & $\begin{array}{c}\text { Exploración de } \\
\text { otras prácticas } \\
\text { profesionales } \\
\text { relacionadas } \\
\text { con el uso de } \\
\text { datos como un } \\
\text { recurso para el } \\
\text { aprendizaje. }\end{array}$ & $\begin{array}{l}\text { Exploración } \\
\text { de otras } \\
\text { actividades de } \\
\text { enseñanza y } \\
\text { aprendizaje } \\
\text { utilizando datos. }\end{array}$ & $\begin{array}{c}\text { Exploración del } \\
\text { significado de } \\
\text { los datos en } \\
\text { la evaluación } \\
\text { tradicional }\end{array}$ & $\begin{array}{c}\text { Exploración } \\
\text { de estrategias } \\
\text { centradas } \\
\text { en el alumno } \\
\text { para promover } \\
\text { prácticas } \\
\text { basadas en } \\
\text { datos }\end{array}$ & $\begin{array}{l}\text { Alentar a los } \\
\text { alumnos a } \\
\text { comprender } \\
\text { los datos en } \\
\text { la disciplina } \\
\text { enseñada }\end{array}$ \\
\hline 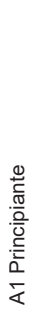 & $\begin{array}{l}\text { Conocimiento } \\
\text { inicial de } \\
\text { prácticas } \\
\text { basadas } \\
\text { en datos, } \\
\text { incertidumbre, } \\
\text { uso básico. }\end{array}$ & $\begin{array}{l}\text { Conocimiento } \\
\text { inicial de los } \\
\text { datos como } \\
\text { posible recurso, } \\
\text { incertidumbre, } \\
\text { uso básico. }\end{array}$ & $\begin{array}{l}\text { Conocimiento } \\
\text { inicial del } \\
\text { problema de los } \\
\text { datos dentro de } \\
\text { la enseñanza y } \\
\text { el aprendizaje, } \\
\text { incertidumbre, } \\
\text { uso básico. }\end{array}$ & $\begin{array}{l}\text { Conocimiento } \\
\text { inicial del } \\
\text { problema de } \\
\text { los datos en } \\
\text { evaluación, } \\
\text { incertidumbre, } \\
\text { uso básico. }\end{array}$ & $\begin{array}{c}\text { Conciencia } \\
\text { sobre la } \\
\text { necesidad de } \\
\text { capacitar a los } \\
\text { estudiantes } \\
\text { para que } \\
\text { comprendan y } \\
\text { utilicen datos } \\
\text { relacionados } \\
\text { con el } \\
\text { aprendizaje y la } \\
\text { vida personal }\end{array}$ & $\begin{array}{c}\text { Conciencia de } \\
\text { la necesidad } \\
\text { de integrar la } \\
\text { alfabetización } \\
\text { en datos en } \\
\text { la disciplina } \\
\text { enseñada, con } \\
\text { incertidumbre y } \\
\text { uso básico. }\end{array}$ \\
\hline
\end{tabular}

Fuente. Adaptado de Redecker y Punie (2017, p.31).

\section{Discusión y Conclusiones}

En este artículo hemos revisado una serie de cuestiones y problemas relacionados con la alfabetización en datos como sector emergente de la alfabetización digital, poniendo particular atención al problema del desarrollo profesional del profesorado universitario en este tema.

Las diversas iniciativas educativas y las tendencias aquí analizadas muestran un panorama de actividad intensa que busca responder a varios objetivos de innovación productiva, organizativa y político-social. Precisamente debido a las múltiples líneas analizadas, podemos concluir que hasta el día de hoy, no existe un enfoque pedagógico sistemático que sea capaz de cubrir las brechas de capacitación de manera programática y tendiente a unir las 
diversas piezas del rompecabezas actual. En este sentido, parece necesario un mayor esfuerzo de elaboración conceptual, que solo puede ser el resultado del trabajo de una comunidad educativa reflexiva, así como el cuidadoso acompañamiento de la investigación educativa sobre nuevas alfabetizaciones. No partimos de una falta de atención al tema de la alfabetización en datos que se ha abierto inmediatamente en relación a la demanda de una sociedad cambiante, y en particular en relación a la ciencia de datos, sino de una fragmentación de las líneas de acción y pensamiento, que impacta de manera particular en el profesorado universitario. Es evidente que las orientaciones de aprendizaje profesional relacionadas con los procesos de «datificación» se han ubicado a nivel de investigación y desarrollo, sea en el ámbito académico o industrial, donde la ciencia de datos nace y toma su forma, sea en cursos de postgrado universitarios avanzados como másteres y doctorados, donde se puede enseñar la ciencia de datos desde un punto de vista técnico avanzado y en conexión con el avance industrial sobre el tema.

Sin embargo, como puede verse fácilmente, este tipo de enfoque está basado sobre las lógica orientadas por las necesidades del mercado, que pueden o no guiar los enfoques razonados y escalables. Como lo demuestra ampliamente el sector de la tecnología educativa, las convocatorias de propuestas y proyectos a menudo crean movimientos de entusiastas y vanguardistas, y con menos frecuencia, la integración o acciones cotidianas que, sin embargo, son las que transforman el sistema y atienden a necesidades inclusivas y de equidad social. Es decir, extender la alfabetización en datos a niveles generales, de transversalidad disciplinar y desde un enfoque socio-técnico de análisis de la relación entre infraestructura tecnológica y práctica social, cultural y política, es aún un ámbito sobre el que se puede hipotetizar un amplio impacto en la población general, y requiere por lo tanto ser explorado. También lo es la forma de integración de los datos en los procesos de enseñanza y aprendizaje, más para el mejoramiento de la comprensión y negociación más participativa sobre los mismos, que para abandonar el alumno a sí mismo etiquetando tal acción como "aprendizaje autoregulado". Es claro que el desarrollo profesional de profesorado universitario, a partir de procesos reflexivos y de 
experimentación que encierran formas de aprendizaje profesional no-formal e informal, puede sentar las bases para enfoques más complejos de la alfabetización básica en datos del estudiantado, que a su vez transferirá estas habilidades a los respectivos contextos de práctica profesional. La elaboración de un marco de referencia para el desarrollo de la alfabetización en datos en el profesorado requiere otras fases de trabajo como un análisis empírico de carácter cualitativo (entrevistas para la validación de componentes y descriptores relevantes en la competencia profesional) y cuantitativo (inventarios con análisis estadístico y extracción de factores o componentes principales). Sin embargo, la base conceptual adoptada, fuertemente basada en la revisión de la literatura y en la adaptación de un marco conceptual existente más amplio (DigCompEdu) se presentan como una base bastante sólida para la tarea.

La discusión deberá necesariamente moverse sobre las herramientas de análisis y promoción de la formación de habilidades básicas y avanzadas en relación con la alfabetización de datos, también vinculada a las epistemologías subyacentes a las formas de conocimiento que ya no se basan en la "recolección", sino en la extracción y la minería de datos. Este último enfoque implica nuevas formas de evaluación y comprensión de los límites de lo dado como un elemento ontológico, como una entidad que en su configuración asume aspectos conceptuales, ideológicos e incluso de poder.

Estas son fronteras de acción y reflexión que no deben olvidarse en los enfoques pragmáticos, a menudo muy orientados al resultado esperado por el mercado y poco basados en la profunda reflexión educativa sobre qué competencias y para qué contextos de desarrollo, o sea, no sólo los tecnológicos e industriales, sino también los sociales y culturales. En particular, en este sentido, vale la pena detenerse en la tesis que expone que la alfabetización de datos viene a solicitar una atención particular a la definición de los datos como elemento contextualizado, donde las matemáticas, las estadísticas y la informática son mediadores eficaces de procesos más profundos, propios del ser humano. En este punto, 
uno podría preguntarse si esta es la oportunidad de salir de la lucha entre las dos culturas que fuera denunciada por el físico y escritor Charles Snow (1959[1964]). No es una prevalencia de ciencias duras o STEM (Science, Technology, Engineering, Math-Ciencia, tecnología, ingeniería y matemáticas-), no es una prevalencia de la esfera humanista-social, sino un diálogo fructífero que permita la generación de interpretaciones (datos) que a su vez apoyen la comprensión del complejo y rico desarrollo de la cultura humana.

\section{Referencias bibliográficas}

Atenas, J., Havemann, L. y Priego, E. (2015). Open Data as Open Educational Resources: Towards Transversal Skills and Global Citizenship. Open Praxis, 7(4), 377-389. https://doi.org/10.5944/openpraxis.7.4.233

Boyer, E. L. (1990). Scholarship reconsidered: priorities of the professoriate (Vol. 1997). San Francisco, CA: Carnegie Foundation for the Advancement of Teaching.

Carlson, J., Fosmire, M., Miller, C. C. y Nelson, M. S. (2011). Determining Data Information Literacy Needs: A Study of Students and Research Faculty. Portal: Libraries and the Academy, 11(2), 629-657. https://doi. org/10.1353/pla.2011.0022

Constantino, G. D. y Raffaghelli, J. E. (2016). Perspectives on Digital Scholarship: In A. Esposito (Ed.), Research 2.0 and the Impact of Digital Technologies on Scholarly Inquiry (pp. 69-96). IGI Global. https://doi. org/10.4018/978-1-5225-0830-4.ch005

Constantino, G. D., Raffaghelli, J. E. y Teijeiro, M. del C. (2015). Profesión Académica Digital (Digital Scholarship): transformaciones del trabajo académico en clave de redes sociales. In Virtual Educa 2015 (pp. 1-15). Acceso en: http://www.virtualeduca.pro/documentos/23/PonenciaConstantino, Raffaghelli y Teijeiro VIRTUAL EDUCA 2015 v.f..pdf 
Daniel, B. (2015). Big Data and analytics in higher education: Opportunities and challenges. British Journal of Educational Technology, 46(5), 904-920. https://doi.org/10.1111/bjet.12230

Daniel, B. K. (2017). Big Data in Higher Education: The Big Picture. In Big Data and Learning Analytics in Higher Education (pp. 19-28). Cham: Springer International Publishing. https://doi.org/10.1007/978-3-31906520-5_3

Dunlap, K. y Piro, J. S. (2016). Diving into data: Developing the capacity for data literacy in teacher education. Cogent Education, 3(1). https:// doi.org/10.1080/2331186X.2015.1132526

EMC Education Services. (2015). Data Science y Big Data Analytics. Indianapolis, IN, USA: John Wiley \& Sons, Inc. https://doi. org/10.1002/9781119183686

European Commission. (2007). Key Competences for Lifelong Learning. European Reference Framework. Luxemburg. Acceso en: http:// ec.europa.eu/dgs/education_culture/publ/pdf/Il-learning/keycomp_ en.pdf

Ferguson, R. (2012). Learning analytics: drivers, developments and challenges. International Journal of Technology Enhanced Learning, 4(5/6), 304-317. Acceso en: http://oro.open.ac.uk/36374/1/ IJTEL40501_Ferguson Jan 2013.pdf

Goodfellow, R. (2014). Scholarly, digital, open: an impossible triangle? Research in Learning Technology, 21. https://doi.org/10.3402/rlt. v21.21366

Hey, A. J. G. (2009). The fourth paradigm: data-intensive scientific discovery. Microsoft Research.

Kennedy, H., Poell, T. y van Dijck, J. (2015). Data and agency. Big Data \& Society, 2(2), 205395171562156. https://doi. org/10.1177/2053951715621569

Kitchin, R. (2014). Big Data, new epistemologies and paradigm shifts. Big Data \& Society, 1(1). https://doi.org/10.1177/2053951714528481 
Kitchin, R. (2015). The Data Revolotion: Big Data, Open Data, Data Infrastructures and Their Consequences (Vol. 1). London: Sage. https:// doi.org/10.1017/CBO9781107415324.004

Knight, S., Buckingham Shum, S. y Littleton, K. (2014). Epistemology, Assessment, Pedagogy: Where Learning Meets Analytics in the Middle. Journal of Learning Analytics, 1(2), 23-47. Li, J., Greenhow, C. y Askari, E. (2016). Scholars in the Digital Age. In Research 2.0 and the Impact of Digital Technologies on Scholarly Inquiry (Vol. 36, pp. 1-16). IGI Global. https://doi.org/10.4018/978-1-5225-0830-4.ch001

Lupton, M. y Bruce, C. S. (2010). Windows on information literacy worlds: Generic, situated and transformative perspectives. In A. Lloyd y S. Talja (Eds.), Practicing information literacy: Bringing theories of learning, practice and information literacy together (pp. 4-27). Wagga-Wagga: Centre for Information Studies, Charles Sturt University.

Manca, S., Caviglione, L. y Raffaghelli, J. E. (2016). Big data for social media learning analytics: potentials and challenges. Journal of E-Learning and Knowledge Society, 12(2). 27-39.

Manca, S. y Ranieri, M. (2017). Exploring Digital Scholarship. A Study on Use of Social Media for Scholarly Communication among Italian Academics. In A. Esposito (Ed.), Research 2.0 and the Impact of Digital Technologies on Scholarly Inquiry (pp. 117-142). Hershey, PA: IGI Global. https://doi.org/10.4018/978-1-5225-0830-4.ch007

Mandinach, E. B. (2012). A Perfect Time for Data Use: Using Data-Driven Decision Making to Inform Practice. Educational Psychologist, 47(2), 7185. https://doi.org/10.1080/00461520.2012.667064

Mandinach, E. B. y Gummer, E. S. (2016). What does it mean for teachers to be data literate: Laying out the skills, knowledge, and dispositions. Teaching and Teacher Education, 60, 366-376. https://doi.org/10.1016/j. tate.2016.07.011

Markham, A. N. (2018). Critical Pedagogy as a Response to Datafication. Qualitative Inquiry, 107780041880947. https://doi. org/10.1177/1077800418809470 
Maybee, C. y Zilinski, L. (2015). Data informed learning: A next phase data literacy framework for higher education. Proceedings of the Association for Information Science and Technology, 52(1), 1-4. https:// doi.org/10.1002/pra2.2015.1450520100108

Nunn, S., Avella, J. T., Kanai, T. y Kebritchi, M. (2016). Learning Analytics Methods, Benefits, and Challenges in Higher Education: A Systematic Literature Review. Online Learning, 20(2). https://doi.org/10.24059/olj. v20i2.790

O'Neil, C. (2016). Weapons of math destruction : how big data increases inequality and threatens democracy. New York: Penguin.

Owen, R., Macnaghten, P. y Stilgoe, J. (2012). Responsible research and innovation: From science in society to science for society, with society. Science and Public Policy, 39(6), 751-760. https://doi.org/10.1093/ scipol/scs093

Pangrazio, L. y Selwyn, N. (2018). 'Personal data literacies': A critical literacies approach to enhancing understandings of personal digital data. New Media y Society, early view. https://doi. org/10.1177/1461444818799523

Pearce, N., Weller, M., Scanlon, E. y Kinsley, S. (2010, December 12). Digital Scholarship Considered: How New Technologies Could Transform Academic Work. In Education. Retrieved from http://ineducation.ca/ ineducation/article/view/44/508

Perrotta, C. y Williamson, B. (2018). The social life of Learning Analytics: cluster analysis and the 'performance' of algorithmic education. Learning, Media and Technology, 43(1), 3-16. https://doi.org/10.1080 /17439884.2016.1182927

Persico, D. y Pozzi, F. (2015). Informing learning design with learning analytics to improve teacher inquiry. British Journal of Educational Technology, 46(2), 230-248. https://doi.org/10.1111/bjet.12207

Prinsloo, P. y Slade, S. (2017). An elephant in the learning analytics room. In Proceedings of the Seventh International Learning Analytics \& Knowledge Conference on - LAK'17 (pp. 46-55). New York, New York, USA: ACM Press. https://doi.org/10.1145/3027385.3027406 
Raffaghelli, J. E. (2017a). Alfabetizzare ai dati nella società dei big e open data: una sfida formativa. Formazione\&Insegnamento - European Journal of Research on Education and Teaching, 25(3), 279-304. https:// doi.org/107346/-fei-XV-03-17_21

Raffaghelli, J. E. (2017b). Exploring the (missed) connections between digital scholarship and faculty development: a conceptual analysis. International Journal of Educational Technology in Higher Education, 14(1), 20. https://doi.org/10.1186/s41239-017-0058-x

Raffaghelli, J. E. (2018a). Educators' Data Literacy Supporting critical perspectives in the context of a "datafied" education. In M. Ranieri, L. Menichetti y M. Kashny-Borges (Eds.), Teacher education \& training on ict between Europe and Latin America (pp. 91-109). Roma: Aracné. https://doi.org/10.4399/97888255210238

Raffaghelli, J. E. (2018b). Oltre il "far di conto" nell'era digitale. La frontiera della data literacy. In M. Ranieri (Ed.), Teoria e pratica delle new media literacies (pp. 99-133). Milano: Aracné. https://doi. org/10.4399/97888548940444

Raffaghelli, J. E., Cucchiara, S., Manganello, F. y Persico, D. (2016). Different views on Digital Scholarship: separate worlds or cohesive research field? Research in Learning Technology, 24(0), 1-17. https:// doi.org/10.3402/rlt.v24.32036

Raffaghelli, J. E. y Manca, S. (2019). Is There a Social Life in Open Data? The Case of Open Data Practices in Educational Technology Research. Publications 2019, Vol. 7, Page 9, 7(1), 9. https://doi.org/10.3390/ PUBLICATIONS7010009

Redecker, C. y Punie, Y. (2017). European Framework for the Digital Competence of Educators: DigCompEdu. Joint Research Centre (JRC) Science for Policy report. https://doi.org/10.2760/159770

Ridsdale, C., Rothwell, J., Smit, M., Ali-Hassan, H., Bliemel, M., Irvine, D., ... Wuetherick, B. (2015). Strategies and Best Practices for Data Literacy Education. https://doi.org/ 10.13140/RG.2.1.1922.5044 
Roll, I. y Winne, P. H. (2015). Understanding, evaluating, and supporting self-regulated learning using learning analytics. Journal of Learning Analytics, 2(1), 7-12. https://doi.org/10.18608/jla.2015.21.2

Scott, A. (2014). Open data for economic growth. 1-20 Washington: World Bank.

Slade, S. y Prinsloo, P. (2013). Learning Analytics, Ethical Issues and Dilemmas. American Behavioral Scientist, 57(10), 1510-1529. https:// doi.org/10.1177/0002764213479366

Slavin, R. E. (2002). Evidence-Based Education Policies: Transforming Educational Practice and Research. Educational Researcher, 31(7), 1521. https://doi.org/10.2307/3594400

Snow, C. (1964). Le due culture (trad. it di). Milano: Feltrinelli.

Stephenson, E. y Schifter Caravello, P. (2007). Incorporating data literacy into undergraduate information literacy programs in the social sciences. Reference Services Review, 35(4), 525-540. https://doi. org/10.1108/00907320710838354

Stewart, B. (2015). Scholarship in Abundance: Influence, Engagement, and Attention in Scholarly Networks. https://doi.org/10.1017/ CBO9781107415324.004

Stewart, B. E. (2013). Massiveness + Openness $=$ New Literacies of Participation? Journal of Online Learning and Teaching, 9(2), 228-238. Acceso en: http://jolt.merlot.org/vol9no2/stewart_bonnie_0613.htm

Stewart, B. E. (2015). Open to influence: what counts as academic influence in scholarly networked Twitter participation. Learning, Media and Technology, 40(3), 287-309. https://doi.org/10.1080/17439884.20 15.1015547

Veletsianos, G. (2013). Open practices and identity: Evidence from researchers and educators' social media participation. British Journal of Educational Technology, 44(4), 639-651. https://doi.org/10.1111/ bjet. 12052 
Veletsianos, G. y Kimmons, R. (2012). Networked Participatory Scholarship: Emergent techno-cultural pressures toward open and digital scholarship in online networks. Computers \& Education, 58(2), 766-774. https://doi.org/10.1016/j.compedu.2011.10.001

Veletsianos, G. y Stewart, B. E. (2016). Discreet Openness: Scholars Selective and Intentional Self-Disclosures Online. Social Media + Society, 2(3), 2056305116664222. https://doi.org/10.1177/2056305116664222

Vuorikari, R., Ferguson, R., Brasher, A., Clow, D., Cooper, A., Hillaire, G., ... Rienties, B. (2016). Research Evidence on the Use of Learning Analytics. Brussels> Joint Research Center. Acceso en: https://doi. org/10.2791/955210

Wasson, B., Hansen, C. y Netteland, G. (2016). Data Literacy and Use for Learning when using Learning Analytics for Learners. In S. Bull, B. M. Ginon, J. Kay, M. D. Kickmeier-Rust y M. D. Johnson (Eds.), Learning Analytics for Learners, 2016 workshops at LAK (pp. 38-41). Edimburg: CEUR. Acceso en http://ceur-ws.org/Vol-1596/paper6.pdf

Williamson, B. (2018). The hidden architecture of higher education: building a big data infrastructure for the 'smarter university.' International Journal of Educational Technology in Higher Education, 15(1), 12. https://doi.org/10.1186/s41239-018-0094-1

Zee, T., van der y Reich, J. (2018). Open Education Science. AERA Open, 4(3), 233285841878746. https://doi.org/10.1177/2332858418787466 